\title{
Durvalumab Consolidation Treatment after Chemoradiotherapy for an HIV-Positive Patient with Locally Advanced Non-Small Cell Lung Cancer
}

\author{
Shoko Kawai ${ }^{a}$ Hiroe Suzukia, busuke Okuma ${ }^{a, c}$ \\ aDepartment of Thoracic Oncology and Respiratory Medicine, Tokyo Metropolitan Cancer \\ and Infectious Diseases Center Komagome Hospital, Tokyo, Japan; 'bepartment of \\ Respiratory Medicine, Tokyo Metropolitan Tama Medical Center, Tokyo, Japan; \\ 'Department of Thoracic Oncology, National Cancer Center Hospital, Tokyo, Japan
}

\section{Keywords}

Locally advanced non-small cell lung cancer - Immune checkpoint inhibitor .

Anti-programmed cell death ligand-1 agent · Human immunodeficiency virus infection

\section{Abstract}

Due to antiretroviral therapy, human immunodeficiency virus (HIV) patients and non-HIV patients have a similar life expectancy. The leading cause of death among HIV patients is lung cancer. However, clinical toxicities with immune checkpoint inhibitors, including durvalumab, in HIV-positive patients with non-small cell lung cancer (NSCLC) remain unknown. We report a 45-year-old Japanese HIV patient, who was safely treated with durvalumab consolidation therapy after concurrent chemoradiotherapy (CCRT) for locally advanced NSCLC without significant toxicities until his disease progressed. This case demonstrates the safety of durvalumab consolidation therapy for HIV-positive patients after CCRT for locally advanced NSCLC.

(c) 2020 The Author(s).

Published by S. Karger AG, Basel

\section{Introduction}

The number of human immunodeficiency virus (HIV)-infected patients with lung cancer has increased, and lung cancer has become a leading cause of death because these patients are less likely to die from acquired immunodeficiency syndrome (AIDS)-related illnesses due to the advent of antiretroviral therapy (ART) [1]. We have limited safety and efficacy data for chemotherapy for advanced non-small cell lung cancer (NSCLC) with a French phase II trial 


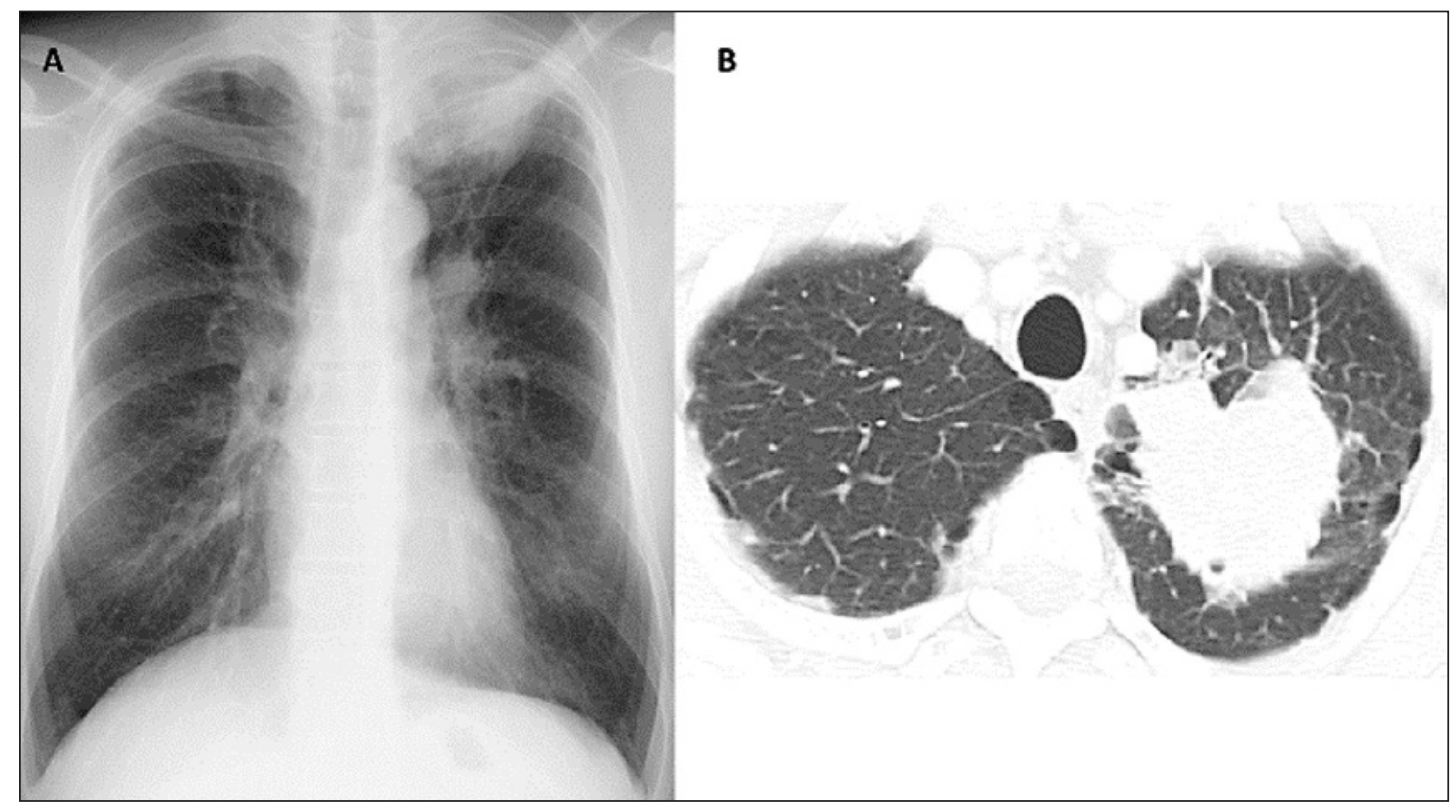

Fig. 1. Radiological findings of a mass in the left upper lobe of the lung. The mass was a locally advanced NSCLC mass. A Chest X-ray. B CT scan.

with a small sample [2], since HIV-positive patients are usually excluded from clinical trials. The safety profiles of immune checkpoint inhibitors (ICIs) are also limited [3,4].

Recently reported cases with ICIs for HIV may work similarly to the so-called shock and kill concept, a hypothesis on the eradication of HIV from patients' bodies [5]. As a result, ICIs became popular not only as a therapy for lung cancer but also as a therapy for HIV infections. For the shock and kill hypothesis, it is important to know whether we can safely use ICIs for lung cancer patients with HIV in the clinical setting.

Herein, we report an HIV-positive patient with lung cancer who was safely treated, including in regard to immune-mediated adverse events (imAEs), with durvalumab.

\section{Case Report}

A 45-year-old male patient had been diagnosed with HIV after being admitted to another hospital because of his iliac fracture at the age of 34 years. However, he did not visit the hospital he was referred to. One year after this, he was arrested for using some recreational drugs. At the age of 37 years, he noticed rashes on his skin, fatigue, and a cough in summer. A few months later, he finally underwent a medical examination at the infectious disease department at our hospital. He was admitted to the hospital because he developed pneumocystis pneumonia and began receiving treatment for AIDS. Following this, his condition stabilized until the age of 45 years.

The lung tumor was found because of his chest pain and cough. His chest computed tomography (CT) indicated a mass measuring approximately $8 \mathrm{~cm}$ in diameter located in the left upper lobe (Fig. 1A, B), and the patient was referred to our department. He was diagnosed with stage IIIB lung adenocarcinoma (Fig. 2A, B). His tobacco history was 18.75 pack-years and he was a current smoker then. Molecular profiling revealed a wild-type epidermal growth factor receptor and was negative for anaplastic lymphoma kinase gene translocation and 


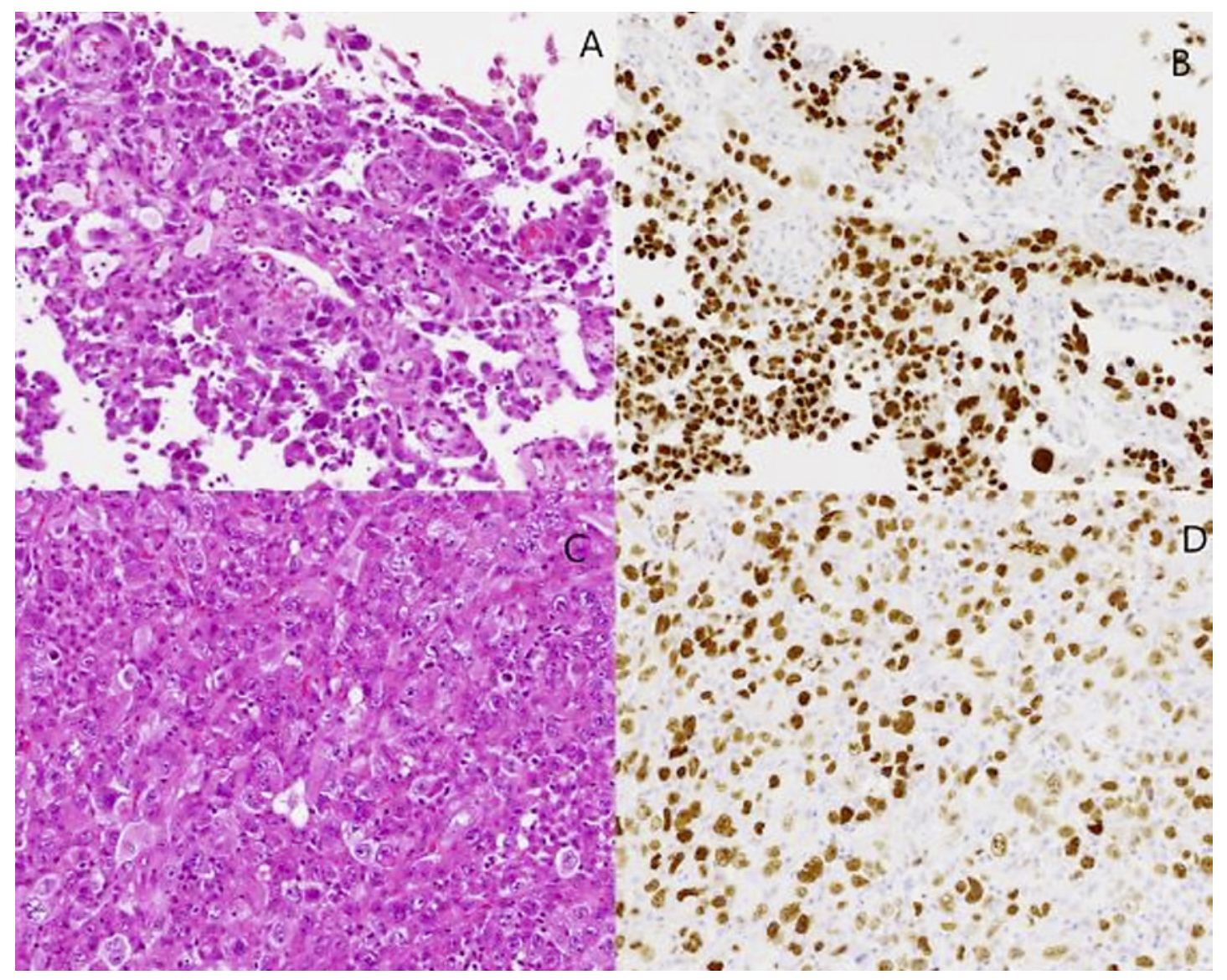

Fig. 2. Hematoxylin and eosin stain of lung tissue showing clusters of malignant cells that were collected before treatment (A). Immunostain for thyroid transcription factor 1 (TTF-1) of lung tissue showing diffuse nuclear staining (B). Hematoxylin and eosin stain of brain metastases after 8 cycles of durvalumab, which shows that the cellular atypia was worse than before. There were only few lymphocytes in those tumor cells (C). Immunostain for TTF-1 of brain metastasis tissue showing diffuse nuclear staining (D).

ROS-1 rearrangement. Programmed death ligand-1 (PD-L1) status (as measured by the 22C3 antibody) was highly expressed (95\%). The patient was treated with weekly carboplatin (CBDCA, area under the curve of 2) + paclitaxel (PTX, $40 \mathrm{mg} / \mathrm{m}^{2}$ ) and concurrent irradiation at a dose of $60 \mathrm{~Gy} / 30 \mathrm{Fr}$ for a primary lesion and mediastinal lymph node involvement. The following treatment-related non-hematological toxicities were noted during treatment: esophagitis (grade 3), nausea (grade 2), and constipation (grade 1). The following hematological toxicities were noted: leukopenia (grade 2), neutropenia (grade 1), and lymphopenia (grade 3). After completion of concurrent chemoradiotherapy (CCRT), a follow-up CT scan showed a reduction in the tumor size (Fig. 3B).

After we confirmed that the esophagitis had improved, durvalumab $(10 \mathrm{mg} / \mathrm{kg})$ consolidation therapy was intravenously administered on day 42 after the last dose of irradiation. Treatment with consolidation durvalumab was continued until the tumor progressed after 4 months. There were no significant imAEs throughout durvalumab treatment. We resected the recurrent brain metastases (Fig. 2C, D; Fig. 3C) and investigated gene mutations with the Oncomine Dx Target Test CDx system. However, none of the 46 genes showed significant genetic alteration. 


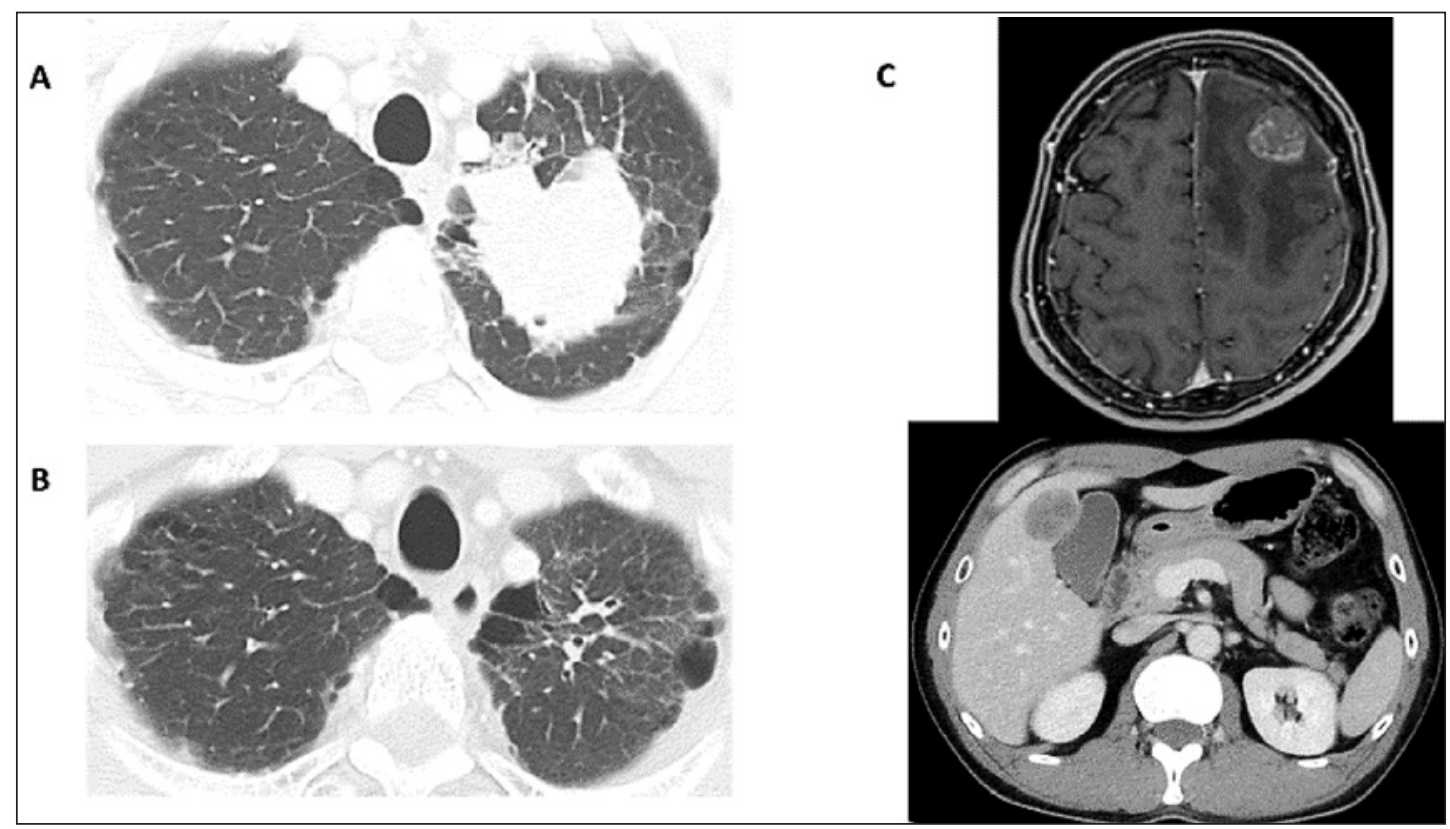

Fig. 3. CT scan during the clinical course. A CT scan before concurrent chemoradiotherapy (CCRT). B A primary lesion of the lung showed a remarkable decrease in size after the completion of CCRT. C Relapsed lesions in the brain and liver after 4 months.

When we found liver metastases after the surgery, the patient was treated with docetaxel $\left(60 \mathrm{mg} / \mathrm{m}^{2}\right)$ and ramucirumab $(8 \mathrm{mg} / \mathrm{kg})$ every 3 weeks as second-line chemotherapy. Six months later, the liver metastases had progressed. The patient was then treated with pemetrexed $\left(500 \mathrm{mg} / \mathrm{m}^{2}\right)$ every 3 weeks as third-line treatment. However, we found the liver metastases became worse after two administrations of pemetrexed. We decided to use TS-1 (60 mg b.i.d.) as the fourth-line treatment. The patient was treated with palliative radiotherapy for liver metastases. He was still treated with weekly gemcitabine $(1,000$ $\mathrm{mg} / \mathrm{m}^{2}$ ).

\section{Discussion}

Several types of cancer have been recognized to be definitively related to immune deficiency in people with AIDS-defining cancer (ADC); lung cancer, as a non-AIDS-defining cancer (NADC), is the most common cancer in this population among all cancers, including ADCs [6]. Advances in ART for HIV patients have allowed a better prognosis for HIV-positive patients [1]. Recently, malignant NADCs became a prognostic factor for patients with HIV [1]. As a result, the issue of chemotherapy for cancer patients with HIV has grown. However, appropriate clinical practice, including optimal dosages, remains uncertain for cancer patients with HIV and fragile bone marrow function [7]. Conversely, it has also been reported that standard therapy for HIV-positive lung cancer patients undergoing ART achieve good outcomes, which are similar to those seen in HIV-negative patients with advanced NSCLC $[8,9]$.

The PACIFIC trial verified the efficacy of durvalumab after CCRT for unresectable locally advanced NSCLC $[10,11]$. ICIs for cancer patients with HIV have been addressed in terms of HIV parameters, and there was no deleterious impact on immunotherapy [3, 4]. However, these studies did not include patients on durvalumab with stage III NSCLC after CRT. 
ICIs are suggested to affect not only lung cancer but also the HIV infection itself [5]. HIV remains in patients' bodies while undergoing ART. HIV-1 remains latent in the form of stopping transcription in a cell group called a reservoir [5]. If ART targets latent HIV cells, we need to reverse the HIV status that stops transcription. Currently, the most-studied approach to minimize latently infected $\mathrm{T}$ cells is based on the hypothesis that the virus or immunemediated cytolysis temporarily activates HIV latency, leading to the annihilation of these cells by the shock and kill concept [5]. In contrast, ICIs may have benefits in treating HIV infection by de-repressing the blockade of HIV transcription in the reservoirs. It has also been suggested that ICIs could restore the function of HIV-1-specific T cells with high PD-L1 expression levels $[12,13]$. Nivolumab eventually decreases patients' viral load, which supports the induced synergistic shock and kill hypothesis [12].

The patient did not show any imAEs from durvalumab consolidation therapy. This indicates that durvalumab after CCRT is a safe treatment for patients with HIV despite the limitation that the safety only applies to Japanese patients in their younger age. However, he also did not demonstrate any effects. A previous study reported that the presence of HIV infection does not appear to be associated with a difference in efficacy of ICI therapy [2]. The objective response rate among HIV-infected patients with NSCLC who received the previous systemic treatment was $26 \%$ and was $50 \%$ among those who did not it, which is similar to findings from major trials of anti-PD-1 inhibitor therapy in patients with NSCLC. However, this patient's treatment was unsuccessful, which demonstrates that the efficacy of ICIs may be questionable in this population. It may either be because of the small number of cases or because of durvalumab for patients with HIV being less effective than other ICIs. In any case, the reason is unknown at this stage.

To reveal the other genetic features of lung cancer, we examined specimens with the Oncomine Dx Target Test CDx system, but there were no genetic alterations in this case. There are limited data regarding the prevalence of HIV mutations in patients with lung cancer [14], which is similar to the data in those without HIV. In addition, there are no driver mutations associated with a poor prognostic outcome for ICIs. In general, there are two types of resistance mechanisms for ICIs: primary resistance and acquired resistance. Recently, it has been suggested that stimulator of interferon genes (STING) is involved in both HIV and cancer immunity. Some research has shown that HIV infection can activate STING, while other research has shown activated STING can increase cancer-specific CD8 ${ }^{+} \mathrm{T}$ cells [15]. If only this immune system is present, it seems that a hypothesis can be made that immune systems in HIV-associated lung cancer patients can adequately suppress their cancers. However, we know HIV-associated advanced NSCLC generally has a poor prognosis. We do not know how these complex immune mechanisms affect the effectiveness of medications. There is no evidence to address why lung cancer patients with HIV have a worse prognosis than other patients.

Prior laboratory and clinical reports suggest that the tissues of patients infected with HIV are more sensitive to radiation therapy [15]. There are many reported cases about mucosal toxicities in HIV patients with Kaposi's sarcoma, and there are some cases of severe esophagitis in patients with lung cancer who underwent irradiation therapy. A previous report from our hospital indicated severe mucositis before a total dose of 20 Gy irradiation was administered to HIV patients with cancer located in the oral cavity and pharynx [15]. The patient in this case experienced odynophagia with only a total dose of 16 Gy irradiation. This symptom rapidly recovered once CCRT was completed. Hence, it is reasonable to conclude that durvalumab was not associated with the severe esophagitis.

In summary, this is the first reported patient to be safely treated with consolidation durvalumab after CCRT as a standard therapy for stage III NSCLC. 


\section{Acknowledgments}

The authors would like to thank Enago (https://www.enago.jp) for the English language review.

\section{Statement of Ethics}

The study was conducted according to the Declaration of Helsinki. With regard to informed consent, the opt-out method (which provides opportunities to target patients for rejection through information disclosure via posting and publication) was employed, without mandating informed consent from individuals for case series. However, it is also affirmed that the subject has given his written informed consent to publish his case in this case.

\section{Disclosure Statement}

The first and second authors have no conflicts of interest to declare. Yusuke Okuma: Astra Zeneca, Boehringer Ingelheim, Takeda Oncology, Chugai Pharmaceutical. Co. Ltd, Ono Pharmaceutical Co. Ltd., MSD Co. Ltd, Ely Lilly.

\section{Funding Sources}

There are no funding sources for this case report.

\section{Author Contributions}

Shoko Kawai and Yusuke Okuma participated in the clinical care of the patient. All authors participated in the manuscript writing and revision. All authors approved the final manuscript to be submitted.

\section{References}

1 Shiels MS, Pfeiffer RM, Gail MH, Hall HI, Li J, Chaturvedi AK, et al. Cancer burden in the HIV-infected population in the United States. J Natl Cancer Inst. 2011;103(9):753-62.

2 Lavole A, Tomasini P, Monnet I, Kiakouama-Maleka L, Quantin X, Taillade L, et al. IFCT-1001 CHIVA trial: a phase II study of carboplatin (Ca) plus pemetrexed (P) followed by P maintenance, as first-line therapy for human immunodeficiency virus (HIV)-associated advanced non-squamous non-small cell lung cancer (NS-NSCLC). J Clin Oncol. 2016;34:9076.

3 Cook MR, Kim C. Safety and efficacy of immune checkpoint inhibitor therapy in patients with HIV infection and advanced-stage cancer: a systematic review. JAMA Oncol. 2019;5(7):1049-54.

4 Ostios-Garcia L, Faig J, Leonardi GC, Adeni AE, Subegdjo SJ, Lydon CA, et al. Safety and efficacy of PD-1 inhibitors Among HIV-positive patients with non-small cell lung cancer. J Thorac Oncol. 2018;13(7):1037-42.

5 Deeks SG, Lewin SR, Ross AL, Ananworanich J, Benkirane M, Cannon P, et al. International AIDS Society global scientific strategy: towards an HIV cure 2016. Nat Med. 2016;22(8):839-50.

6 Grulich AE, van Leeuwen MT, Falster MO, Vajdic CM. Incidence of cancers in people with HIV/AIDS compared with immunosuppressed transplant recipients: a meta-analysis. Lancet. 2007;370(9581):59-67.

7 Sanchez X, Cousins-Hodges B, Aguilar T, Gosselink P, Lu Z, Navarro J. Activation of HIV-1 coreceptor (CXCR4) mediates myelosuppression. J Biol Chem. 1997;272(44):27529-31.

8 Okuma Y, Tanuma J, Kamiryo H, Kojima Y, Yotsumoto M, Ajisawa A, et al. A multi-institutional study of clinicopathological features and molecular epidemiology of epidermal growth factor receptor mutations in lung cancer patients living with human immunodeficiency virus infection. J Cancer Res Clin Oncol. 2015;141(9):1669-78. 
9 Okuma Y, Yanagisawa N, Hosomi Y, Imamura A, Okamura T, Kato K, et al . Concomitant chemoradiotherapy and antiretroviral therapy for HIV-infected patients with locoregionally advanced non-small cell lung cancer: benefit and tolerability of treatment in 2 cases. Onkologie. 2013;36(10):586-90.

10 Antonia SJ, Villegas A, Daniel D, Vicente D, Murakami S, Hui R, et al . PACIFIC Investigators. Durvalumab after chemoradiotherapy in stage III non-small-cell lung cancer. N Engl J Med. 2017;377(20):1919-29.

11 Antonia SJ, Villegas A, Daniel D, Vicente D, Murakami S, Hui R, et al., PACIFIC Investigators. Overall survival with durvalumab after chemoradiotherapy in stage III NSCLC. N Engl J Med. 2018;379:2342-50.

12 Guihot A, Marcelin AG, Massiani MA, Samri A, Soulié C, Autran B, et al. Drastic decrease of the HIV reservoir in a patient treated with nivolumab for lung cancer. Ann Oncol. 2018;29:517-8.

13 Gay CL, Bosch RJ, Ritz J, Hataye JM, Aga E, Tressler RL, et al.; for the AIDS Clinical Trials 5326 Study Team. Clinical trial of the Anti-PD-L1 antibody BMS-936559 in HIV-1 infected participants on suppressive antiretroviral therapy. J Infect Dis. 2017;215(11):1725-33.

14 Thaler J, Sigel C, Beasley MB, Wisnivesky J, Crothers K, Bauml J, et al. Clinically significant mutations in HIVinfected patients with lung adenocarcinoma. Br J Cancer. 2017;117(9):1392-5.

15 Kaminuma T, Karasawa K, Hanyu N, Chang TC, Kuga G, Okano N, et al. Acute adverse effects of radiation therapy on HIV-positive patients in Japan: study of 31 cases at Tokyo Metropolitan Komagome Hospital. J Radiat Res. 2010;51(6):749-53. 